\title{
Full configuration drag estimation of short-to-medium range fixed-wing UAVs and its impact on initial sizing optimization
}

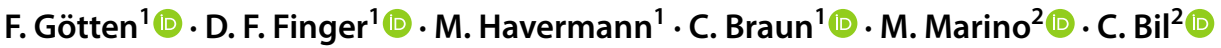

Received: 18 February 2021 / Revised: 21 April 2021 / Accepted: 3 June 2021 / Published online: 10 June 2021

(c) The Author(s) 2021

\begin{abstract}
The paper presents the derivation of a new equivalent skin friction coefficient for estimating the parasitic drag of short-tomedium range fixed-wing unmanned aircraft. The new coefficient is derived from an aerodynamic analysis of ten different unmanned aircraft used for surveillance, reconnaissance, and search and rescue missions. The aircraft is simulated using a validated unsteady Reynolds-averaged Navier Stokes approach. The UAV's parasitic drag is significantly influenced by the presence of miscellaneous components like fixed landing gears or electro-optical sensor turrets. These components are responsible for almost half of an unmanned aircraft's total parasitic drag. The new equivalent skin friction coefficient accounts for these effects and is significantly higher compared to other aircraft categories. It is used to initially size an unmanned aircraft for a typical reconnaissance mission. The improved parasitic drag estimation yields a much heavier unmanned aircraft when compared to the sizing results using available drag data of manned aircraft.
\end{abstract}

Keywords Parasitic drag $\cdot \mathrm{UAV} \cdot \mathrm{CFD} \cdot$ Aircraft sizing

$\begin{array}{ll}\text { Abbreviations } \\ \text { AoA } & \text { Angle of attack } \\ \mathrm{AR} & \text { Aspect ratio } \\ C_{\mathrm{D}} & \text { Drag coefficient } \\ C_{\mathrm{Dmin}} & \text { Minimum drag coefficient } \\ C_{\text {feq }} & \text { Equivalent skin friction coefficient } \\ C_{\mathrm{L}} & \text { Lift coefficient } \\ \text { EARSM } & \text { Explicit algebraic Reynolds stress } \\ \text { EO/IR } & \text { Electro-optical infrared }\end{array}$

F. Götten

goetten@fh-aachen.de

D. F. Finger

f.finger@fh-aachen.de

M. Havermann

havermann@fh-aachen.de

C. Braun

c.braun@fh-aachen.de

M. Marino

matthew.marino@rmit.edu.au

C. Bil

cees.bil@rmit.edu.au

1 Department of Aerospace Engineering, FH Aachen University of Applied Sciences, Aachen, Germany

2 School of Engineering, RMIT University, Melbourne, Australia

$\begin{array}{ll}\text { ICE } & \text { Internal combustion engine } \\ \text { MTOM } & \text { Maximum take-off mass } \\ \text { P/W } & \text { Power-to-weight ratio } \\ \text { Re } & \text { Reynolds number } \\ \text { SIMPLE } & \text { Semi Implicit Method for Pressure Linked } \\ & \text { Equations } \\ \text { SMR } & \text { Short-to-medium range } \\ S_{\text {ref }} & \text { Aircraft reference area } \\ \text { SST } & \text { Shear stress transport } \\ S_{\text {wet }} & \text { Aircraft wetted area } \\ \text { UAV } & \text { Unmanned Aerial Vehicle } \\ \text { URANS } & \text { Unsteady Reynolds Averaged Navier Stokes } \\ \text { W/S } & \text { Wing loading } \\ y^{+} & \text {Normalized wall distance }\end{array}$

\section{Introduction}

Conceptual aircraft design is a multidisciplinary optimization problem. The design routine is divided into several sub-models that are responsible for estimating the new aircraft's properties, including weight, propulsion, cost, or aerodynamics. The accuracy of such underlying models is of critical importance as their outcomes drive the design to convergence. One of these critical models is responsible for estimating the new aircraft's parasitic drag. The 
parasitic drag has a direct effect on flight performance prediction and propulsion system sizing.

A well-known approach is to use an equivalent skin friction method. In this method, the complete parasitic drag of an aircraft is based on an equivalent skin friction coefficient and its multiplication with the wetted area of the airframe. The empirical skin friction coefficient depends on the aircraft category to account for their specific aspects [1]. A careful literature review revealed that neither an equivalent skin friction factor nor wetted area correlations have yet been derived for short-to-medium range UAVs. These UAVs have take-off masses from $15 \mathrm{~kg}$ up to about $700 \mathrm{~kg}$, and they are often used for reconnaissance, surveillance, and search and rescue missions. Both civil and military users employ such UAVs on long endurance missions from about 4-16 h [2, 3]. The relative range of take-off masses is rather significant, which is a characteristic of this UAV class. There is no uniform classification for such UAVs, and multiple ones have been proposed in the past [2]. They are sometimes termed shortrange (SR) to medium-range (MR) UAVs, but also referred to as "tactical UAVs" [4]. For reasons of simplicity, they are named SMR UAVS (short-to-medium range) in this publication.

These UAVs are larger than micro and small UAVs, where aerodynamic aspects are of minor importance, but they are also significantly smaller than Medium-Altitude or High-Altitude Long Endurance (MALE/HALE) UAVs, where manned aircraft drag estimations might be used [5]. In such SMR UAVs can be seen as the lightest UAV class for which aerodynamic aspects are critical, and their sizes and shapes dictate different aerodynamic behaviour compared to heavier aircraft. Prominent examples of this aircraft category are the Aeronautics Aerostar or the AAI Shadow 200. SMR UAVs employed for reconnaissance, surveillance, and search and rescue missions are often designed using the twin-tail boom configuration with a pusher propeller. In this configuration, the exhaust gases of the combustion engine do not interfere with the optical sensors.

The past development of SMR UAVs was very concerned with data-link capabilities, autopilot functionality, and safety aspects. Aerodynamic design was often subordinated, even though SMR UAVs are very sensitive to aerodynamic aspects, including parasitic drag [3]. Only very recently did research interest also extend to the aerodynamic design and optimization of SMR UAVs [6, 7].

Little information about the parasitic drag of SMR UAVs is currently available [8]. UAV designers nowadays rely on available data of other aircraft categories. As SMR UAVs feature particular configurational aspects like solid springtype landing gears or EO/IR sensor turrets, their parasitic drag might not be comparable to these aircraft categories. As such, the drag and flight performance estimation of SMR
UAVs is subject to large uncertainties. This can have a significant effect on the outcomes of a design process.

This publication, therefore, presents new aerodynamic parasitic drag data of 10 representative UAVs. The data is generated using a validated Unsteady Reynolds-averaged Navier-Stokes (URANS) approach. The UAVs are chosen as representatives for a variety of configurations. The aerodynamic data is used to derive a novel equivalent skin friction coefficient that allows a full-configuration drag estimation of SMR UAVs. The new coefficient can be used in combination with geometrical data of an in-house database to directly estimate the drag of a new UAV.

In a parallel research effort, a tool for the initial sizing of general aviation aircraft has been developed [9]. It allows the sizing of conventional, fully-electric, and hybrid-electric aircraft. Recently, the tool has also been expanded to allow the sizing of SMR UAVs. The newly derived drag estimation technique for those aircraft is used in the aerodynamic model of this tool to increase its capabilities. The paper will further show the impact of the enhanced drag estimation accuracy based on the sizing results of an SMR UAV.

\section{Methodology}

\subsection{Equivalent skin friction approach}

The equivalent skin friction method models an aircraft's parasitic drag with a skin friction coefficient in analogy to a flat plate. This equivalent skin friction coefficient is multiplied with the aircraft's total wetted area, which is assumed to be proportional to the total parasitic drag. The basic formulation of the method is given in Eq. (1).

$C_{\text {D min }}=C_{\text {feq }} \cdot \frac{S_{\text {wet }}}{S_{\text {ref }}}$

The application of the method requires an equivalent skin friction coefficient and the ratio of the aircraft's wetted area to reference (wing) area as inputs. Some wetted area estimates depending on the aircraft class are given in Raymer [1]. However, no data for UAVs is available.

The equivalent skin friction coefficient strongly depends on the aircraft category for two distinctive reasons: first, the size and flight speed of the aircraft category dictates its Reynolds number range. Larger aircraft at higher speeds operate at significantly larger Reynolds numbers than small, low-speed aircraft. Generally, an increase in Reynolds number reduces the turbulent skin friction coefficient and, therefore, also the equivalent skin friction coefficient.

Second, the equivalent skin friction coefficient has to represent the complete parasitic drag of an aircraft. However, only a portion of the aircraft's parasitic drag is friction drag 
and thus proportional to the wetted area. A large amount of aircraft parasitic drag can be pressure drag caused by flow separation or bluff body flow. Pressure drag is not proportional to the aircraft's wetted area, and aircraft with significant parasitic pressure drag must have higher equivalent skin friction coefficients to account for this. The amount of parasitic pressure drag depends on the aerodynamic smoothness of the aircraft and, thereby, on the aircraft category.

An overview of equivalent skin friction coefficients for various aircraft categories is found in Ref. [1], and here shown in Table 1. It is important to note that these values are averaged for each aircraft category. The values for individual aircraft might also significantly scatter within a certain aircraft class, see Ref. [10]. This is caused by the individual aerodynamic behaviour of each aircraft. The level of scattering is generally accepted given that the method is only applied in very early aircraft design stages. Therefore, the application of average values is common. To date, no equivalent skin friction coefficient for SMR UAVs is available.

\subsection{UAV geometry selection and modeling}

The UAVs that were investigated in this study were chosen according to the findings of an extensive in-house database. It provides a range of statistical data that includes general dimensions, detailed shapes, and flight performance parameters. Very little information on the detailed geometry of SMR UAVs is openly available as most manufacturers keep these a secret. Therefore, the information for the database was taken from high-quality images or three-view drawings. A central part of the database is the estimation of a UAV's wetted area as a direct input into the equivalent skin friction drag estimation. The geometry is simplified and approximated by shapes for which analytical equations are available. Depending on the component, multiple geometrical representations are available, and the most realistic one can be chosen by the user. The geometrical breakdown of the UAVs is detailed. Taking the data acquisition accuracy into account, the overall accuracy for the total UAV wetted area is about $10-15 \%$. This is considered adequate for the desired purpose. Further information on the database can be found in Ref. [11]

Table 1 Equivalent skin friction coefficients for various aircraft

\begin{tabular}{lll}
\hline Aircraft type & $C_{\text {feq }}$ & $\begin{array}{l}\text { Typical cruise } \\
\text { wing-chord Re }\end{array}$ \\
\hline Jet Bomber and Civil Transport & $0.0026-0.0030$ & $80,000,000$ \\
Military Cargo & 0.0035 & $80,000,000$ \\
Jet Fighter & 0.0035 & $30,000,000$ \\
Light Aircraft-Single Engine Prop & 0.0055 & $8,000,000$ \\
Light Aircraft-Twin Engine Prop & 0.0045 & $12,000,000$ \\
\hline
\end{tabular}

10 SMR UAVs included in the database were selected as being representative, and their geometry remodeled using NASA's OpenVSP [12]. The CAD models of all UAVs were derived from drawings and images and do not pose exact $\mathrm{CAD}$ geometries from manufacturers. While being representative of the analyzed UAV class, one should not treat the presented data as original data of the real aircraft. It is, however, reasonable to assume that the models behave realistically in terms of their level of parasitic drag and the individual drag contribution of each aircraft component.

Table 2 gives an overview of the UAVs' most important data. Take-off masses range from $25 \mathrm{~kg}$ up to $630 \mathrm{~kg}$ and therefore reflect the typical range of SMR UAVs. This range might seem rather large compared to take-off mass ranges of manned aircraft categories but is a characteristic of this class of UAVs [2]. Even though the UAVs show significant size differences, their geometry, mission scenarios, and use cases are quite similar. The UAVs mostly feature the twin tailboom configuration, a fixed tricycle landing gear, and an EO/IR sensor turret attached to their lower fuselage. Aspect ratios are moderate to large, and they are employed for surveillance, reconnaissance or research missions. Such scenarios follow a typical flight profile, which is highlighted in Fig. 1. The UAV takes off, climbs to a designated altitude, and cruises to its surveillance or reconnaissance area. There it loiters for an extensive amount of time, cruises back and lands again. Nominal flight altitudes are on the order of 500-1500 m, and loiter speeds optimized for long endurance [5].

The similar mission scenarios are also reflected in comparable payload fractions, which range from 0.14 to 0.25 for all but the heaviest UAV. This one has a payload fraction of only 0.087 but by far the longest endurance. In such, payload fraction is treated against fuel fraction. Wing aspect ratios are optimized for low speed loiter flight and range from 7.5 up to 16.5. Only the two heaviest UAVs with the longest endurances feature values above 16 , while most of the smaller UAVs have aspect ratios between 7.5 and 10 .

The endurance statements of the UAVs can vary considerably, and care has to be taken when interpreting the data. Manufacturers tend to lengthen their UAVs' endurances for advertisement purposes and sometimes provide values that can only be reasonably achieved, reducing payload capabilities. All analyzed UAVs are shown in Fig. 2. This figure is not to scale and trimmed for visualization purposes. A scaled top-view of the UAVs is presented in Fig. 3. It is recognized that while the typical twin-tailboom configuration is dominant, the exact shapes of the UAVs differ, representing the design philosophies of the manufacturers. These shape differences are also expected to have an influence on the equivalent skin friction coefficient, given their effect on the aerodynamic smoothness of the UAVs. Previous research also highlighted that equivalent skin friction coefficients 


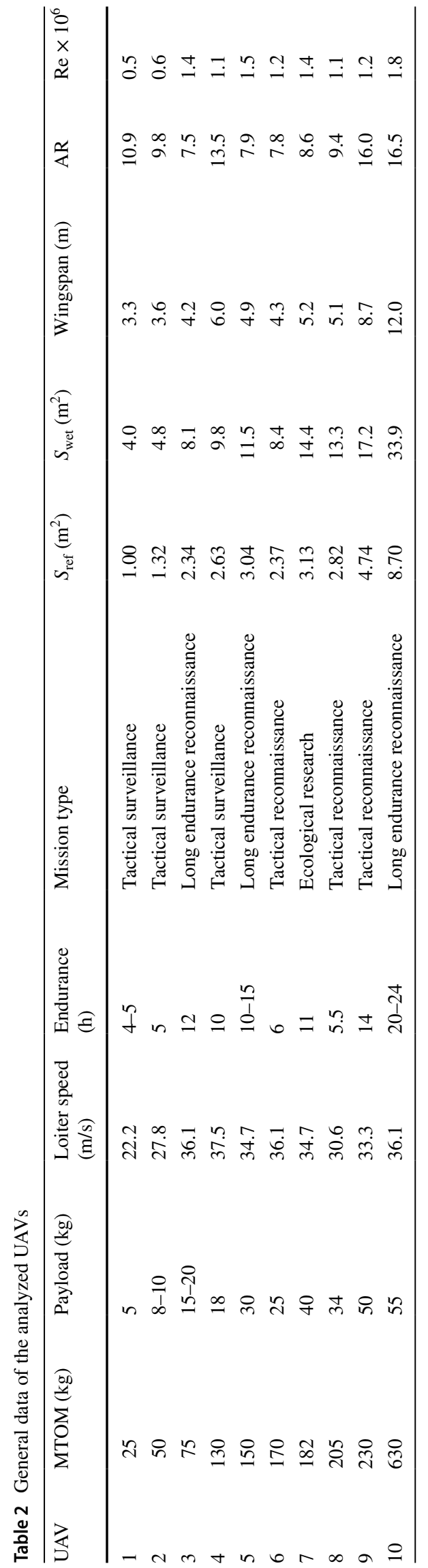

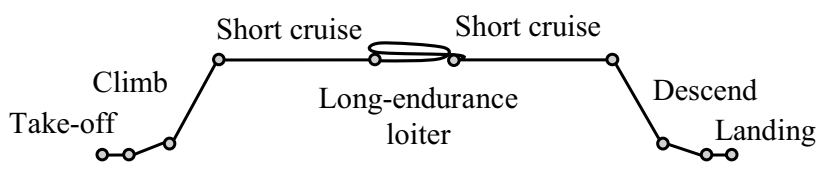

Fig. 1 Typical fligh profile for SMR UAV mission

might significantly scatter within a certain aircraft class [1, 10]. This scatter was generally accepted in the past. Attempts were made to further divide the analyzed UAVs into subcategories to enhance the accuracy of the equivalent skin friction coefficient. However, these did not show promising results mainly for two reasons:

First, the analyzed UAVs already belong to one characteristic UAV class, show similar mission scenarios, and generally a similar aircraft configuration, see Refs. [2, 4, 5]. A further subdivision would require to specify additional criteria on how to distinguish UAVs within the class of SMR UAVs. This might reduce data scatter as it would enhance similarities between the aircraft. However, it also decreases the flexibility of application of the equivalent skin friction coefficient for the entire class of SMR UAVs.

Second, the current sample of ten UAVs is not large enough for separation in subclasses. Further dividing the group of UAVs would result in subclasses that maybe only feature one or two UAVs. This does not allow developing a meaningful statistical basis for the equivalent skin friction coefficient. Further research is required to provide additional drag data for UAVs of the SMR category, allowing a further subdivision.

The choice of the UAVs was also influenced by the availability of three-view drawings and images that allowed accurate modelling. All UAVs feature reciprocating engine propulsion systems. For simplification, aero-propulsive effects were not considered, and the propulsion system was not included in the models. The limited amount of information required some simplifications of the airframe's shapes. The same EO/IR sensor turret shape is attached to the lower fuselage side of each UAV. The EO/IR turret geometry was designed based on another in-house turret database and chosen as being representative for a wide variety of turrets. The positioning and size of the individual turret for each UAV follow the findings of the database.

No information concerning the airfoils used on the wing and tail surfaces were available. For the tail surfaces, symmetric NACA 0010 or NACA 0012 airfoils were chosen depending on the actual thickness of the UAV's tail surface. These airfoils are characteristic of horizontal or vertical stabilizers on a wide variety of aircraft [5].

The formulation of the equivalent skin friction coefficient only includes the parasitic (lift-independent) part of the aircraft's drag. Lift-induced drag is not covered and must be excluded from the findings. One could choose a typical 


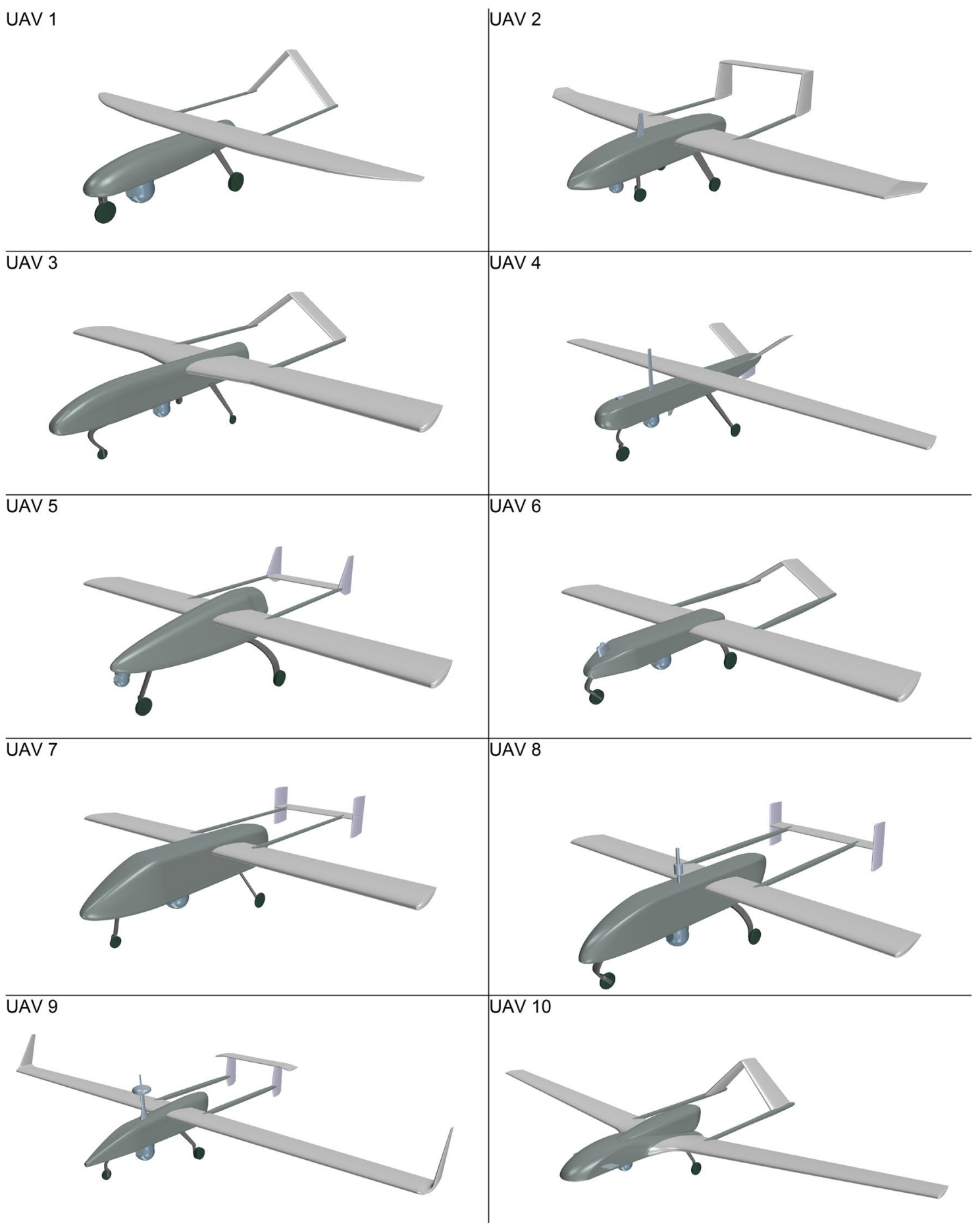

Fig. 2 Isometric views of the analyzed UAVs (not to scale)

cambered airfoil for the UAVs' wings and trim the wing incidence angle to reach the desired lift coefficient. However, in this configuration, the influence of induced drag is significant. To adequately account for the lift-independent drag formulation, the wing's induced drag must be subtracted from the wing's parasitic pressure drag. This, however, is not trivial using a volume resolving CFD method. Lift-induced drag is often calculated by wake surveys that are prone to numerical diffusion effects. Furthermore, a strict distinction between the vortices causing lift-induced drag of the wing and other flow protuberances in the far-field is not directly possible in case a full aircraft configuration is considered. 


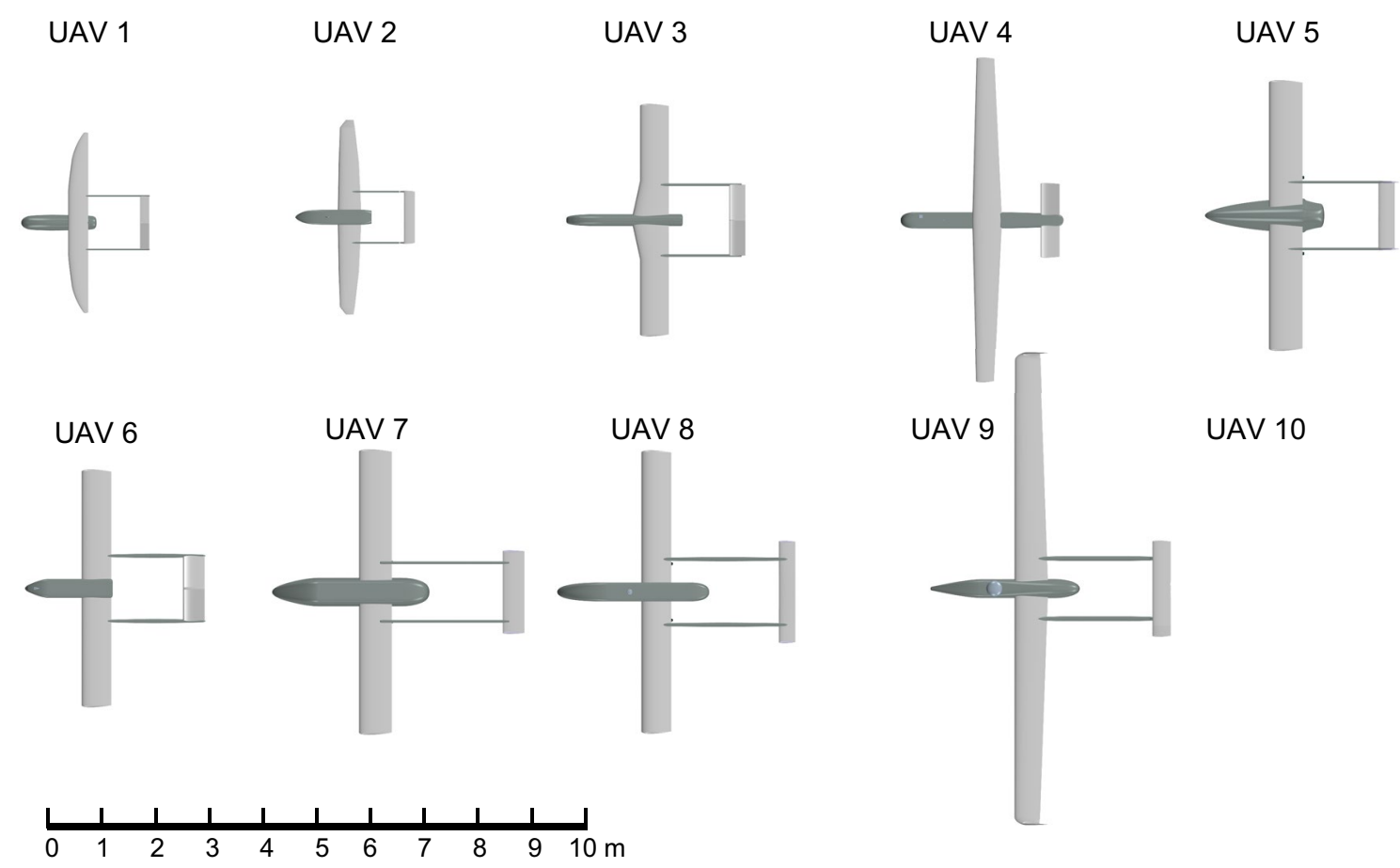

Fig. 3 Top-view of all analyzed UAVs; 1-10 from top left to bottom right (figure to scale)

Further information is provided in Refs. $[13,14]$. These factors significantly reduce the accuracy of the wing-induced drag calculation and therefore enhance the inaccuracy of the wing's parasitic pressure drag computation.

It was therefore decided to employ the symmetric NACA 0015 airfoil over the complete wingspan of each UAV. The symmetric airfoil, in combination with zero wing incidence, automatically enforces zero-lift conditions.

\subsection{Numerical simulation approach}

The analyses were performed using the computational fluid dynamics software StarCCM+v15.02. This finite volume solver is well-known in both industry and academia and has been validated for a variety of cases [15]. The Unsteady Reynolds-averaged Navier-Stokes (URANS) equations are solved with a SIMPLE (Semi Implicit Method for Pressure Linked Equations) algorithm assuming incompressibility due to the low Mach number regime (see Ref. [16]). Both the physical and numerical approach follows the guidelines presented in Ref. [17].

The numerical grid in the free volume was of an unstructured Cartesian type that allows the discretization of arbitrarily complex geometries. The near-wall flow was resolved with a dedicated prismatic boundary layer mesh. The numerical solution was second-order accurate in both space and time. It employed upwind schemes for discretizing convective fluxes, while diffusive ones were approximated with central differences. Menter's Shear Stress Transport (SST) model closed the RANS equations combined with a cubic non-linear constitutive option. This Explicit Algebraic Reynold Stress Model (EARSM) enhances the prediction capabilities of anisotropy of turbulence [18, 19]. It is valuable for secondary flows caused by swirls or separation. The freestream turbulence intensity at the flow inlet was set to $0.5 \%$ in combination with a turbulent viscosity ratio of 5. Sustainment terms added to the transport equations of the turbulence model guaranteed that both turbulent kinetic energy and specific dissipation rate did not dissipate in the free flow [20].

All UAVs were analyzed at zero degrees angle of attack, assuming free atmospheric flight conditions. Therefore, a large flow domain was used that extended 30 times the maximum body length in flow direction and 15 times perpendicular to the flow.

At the inlet boundary conditions, velocity, ambient pressure, and turbulence variables were fixed. The inlet velocity corresponded to the given Reynolds number in Table 2, assuming mean sea level conditions. The pressure at the outlet boundary condition was set to the ambient reference pressure, while the velocity was extrapolated from the adjacent cells in the domain. A cut through the $3 \mathrm{D}$ domain is shown in Fig. 4.

The UAV surfaces were discretized with quadratic cells following guidelines given in Ref. [17]. At least 80 cells were used in the chord-wise direction of the lifting surfaces 


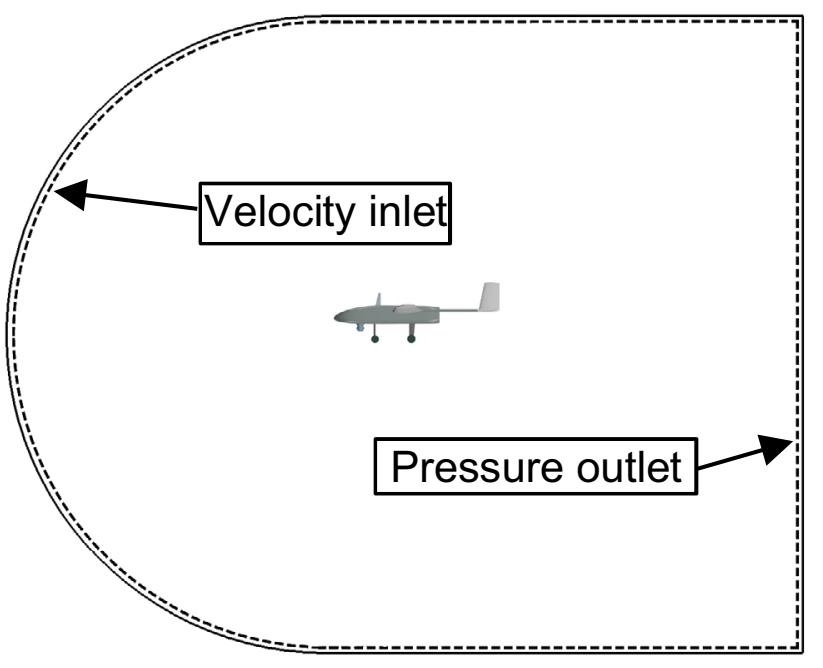

Fig. 4 Simulation domain on the symmetry plane (not to scale)

with additional refinements at leading and trailing edges. The near-wall region was discretized with 30 prismatic cell layers, whereby the first cell height ensured having $y^{+}$values on the order of $0.5-0.8$ for all cases. At least 10 cell layers discretized the viscous sublayer. The transition between the boundary layer mesh and core mesh was smooth, with cell sizes being very similar. The surface mesh of UAV 9 is shown in Fig. 5, together with a close-up of the boundary layer and volume mesh.

The overall cell count of each simulation depends on the individual UAV and its complexity. It varies between 55 and 80 million hexahedral cells. Grid dependency studies were conducted for all UAV configurations ensuring grid-independent results for the total drag and the drag of individual components. Exemplary grid dependency studies of UAVs 1 and 10 showing total drag and turret drag are presented in Fig. 6.

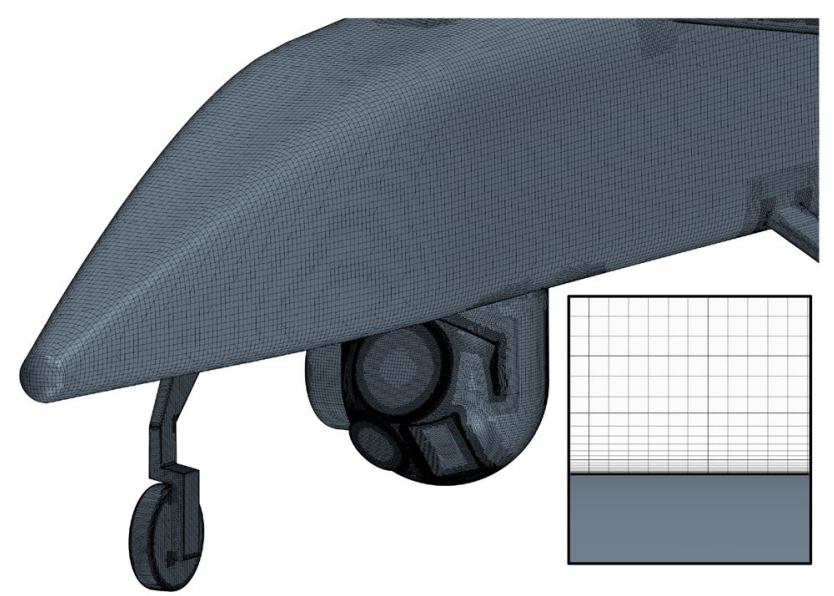

Fig. 5 Level of surface discretization and boundary layer mesh

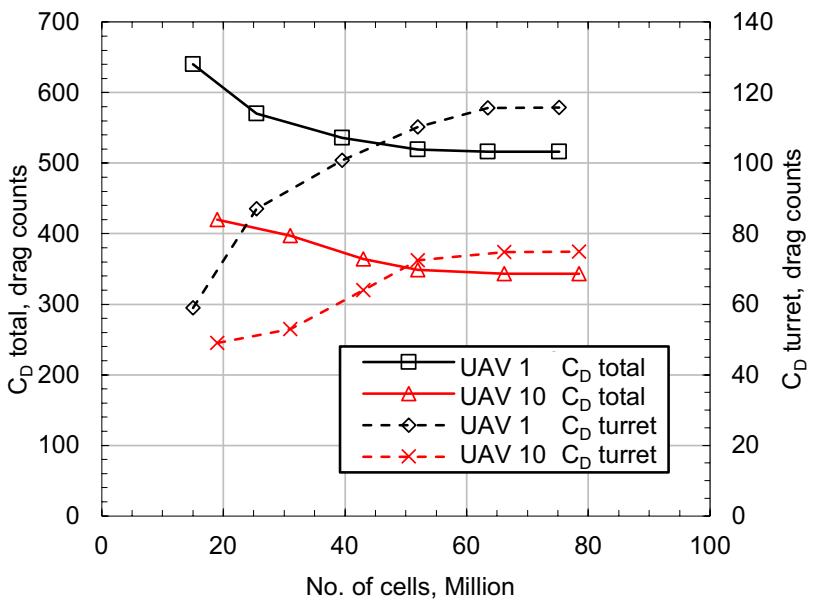

Fig. 6 Grid dependency study for UAVs 1 and 10

The time step was approximated with typical Strouhal numbers of spheres and cylinders for comparable Reynolds numbers [21]. It was later adjusted by testing several time step reductions, whereby a value of $2.5 \times 10^{-4} \mathrm{~s}$ was found to be optimal. After initial stabilization, the simulations were run for at least two more seconds (8000 time-steps). This corresponds to about $60-120$ vortex oscillation periods of spheres or cylinders at comparable Reynolds numbers.

\subsection{Validation}

The simulation approach is validated by comparing it to a wind tunnel study of an 0.4-scale model of the AAI RQ-2 Pioneer UAV presented by Bray in Ref. [22]. The Pioneer UAV is a $205 \mathrm{~kg}$ surveillance and reconnaissance UAV employed by the United States and Israel. The model was tested in the Low-Speed Wind-Tunnel (LSWT) at Wichita State University. The tests were run at a chord-based Reynolds number of about 1.06 million, which corresponds to the full-scale free flight Reynolds number in 10,000 ft. The wing of the wind tunnel model has a NACA 4415 airfoil section and an incidence of two degrees. In such, minimum drag conditions are expected for positive lift. The model features a dummy payload consisting of a hemisphere attached to the lower fuselage. Further geometrical details of the model are presented in Ref. [22]. An angle of attack sweep from $-8^{\circ}$ up to $14^{\circ}$ was simulated with the above presented unsteady RANS approach. The results for both lift, drag, and moment coefficients are given in Figs. 7 and 8. Both lift, drag, and longitudinal moment match the wind tunnel data within 5\% for moderate angles of attack. The simulation overpredicts the lift coefficient at higher angles of attack, which is typical for RANS-based approaches. However, stall conditions are achieved earlier than in the wind tunnel. 


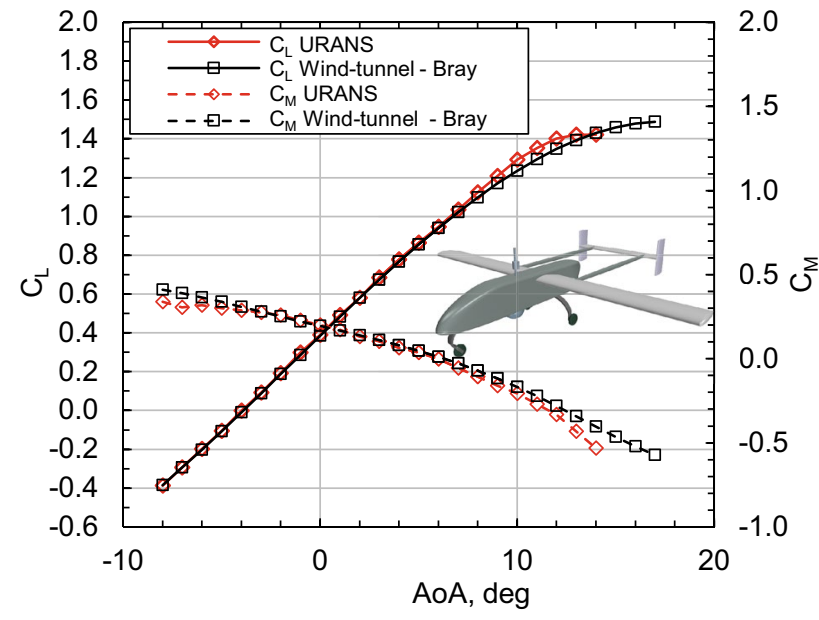

Fig. 7 Pioneer lift and moment with data from Ref. [22]

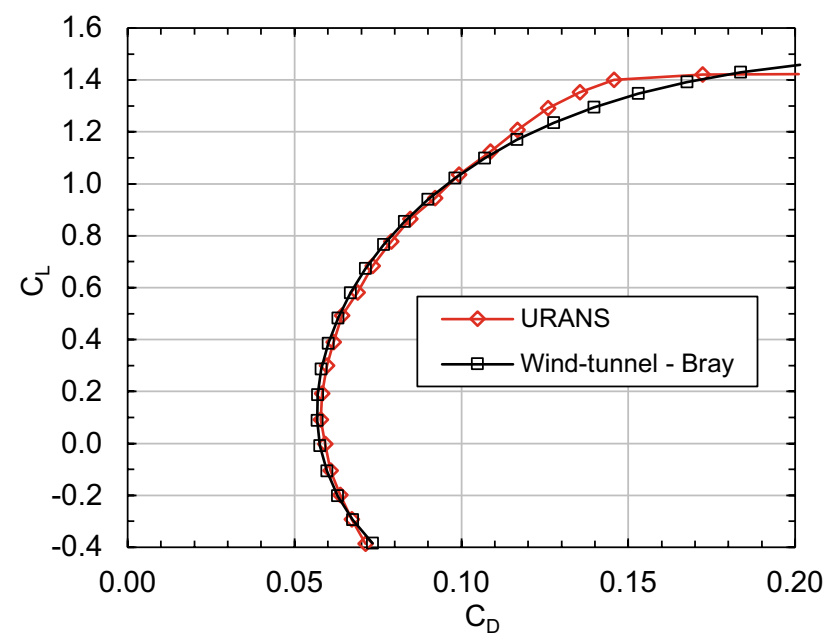

Fig. 8 Pioneer drag polar with data from Ref. [22]

The longitudinal moment matches the wind tunnel data for moderate angles of attack and starts deviating when approaching stall conditions. Drag is accurately simulated up to about 8 degrees angle of attack $\left(C_{\mathrm{L}}=1.0\right.$, deviations below 5\%).

Higher lift conditions show an underestimation of drag along with an overprediction of lift. The validation highlights that the chosen simulation approach is sufficiently accurate for the desired analysis cases.

\section{Results}

The following section describes the results of the numerical analyses. A full aerodynamic investigation of the UAVs is beyond the scope of this publication. Therefore, only an overview of the pressure coefficient distribution on each of the UAVs' surfaces is given in Fig. 9. Further investigations focus on the parasitic drag of the UAVs.

Stagnation conditions are always found at the fuselage noses, while only a moderate level of flow acceleration is noted on the wing's surfaces. This is due to the chosen symmetric airfoil and zero degrees angle of attack. Significant flow acceleration and separation effects can be seen for both the landing gears and EO/IR turrets.

\subsection{Parasitic drag analysis}

The lift-independent pressure drag of the UAV's wings is corrected for airfoil camber effects with the following approach: Airfoil drag generally consists of friction and pressure drag. Assuming fully attached flow, the pressure drag is referred to as boundary layer pressure drag. This drag is essentially due to the way the boundary layer changes the viscous pressure distribution of the airfoil compared to the inviscid case. Cambered and symmetric airfoils obviously have different inviscid pressure distributions. The presence of the boundary layer changes their pressure distributions, which yields larger boundary layer pressure drag for cambered airfoils due to their shape and the associated higher levels of flow acceleration [23]. This is valid for small and moderate angles of attack. It increases the total airfoil drag (often termed profile drag) of a cambered airfoil compared to a symmetric one.

The increased profile drag of cambered airfoils manifests itself in an increased lift-independent drag of a 3D wing compared to a wing with a symmetric airfoil. Based on wind-tunnel data from Ref. [24] and own data of the authors, a representative NACA 4415 airfoil used on several UAVs shows 1.08 times higher profile drag compared to a NACA 0015 airfoil assuming fully turbulent flow. The wing's drag is, therefore, increased by this factor. The influence on total drag, is generally small and does not exceed $3 \%$ of the total drag. For consistency, this correction is only applied for developing the equivalent skin friction coefficient and not for the investigation of the drag break-down as derived from the CFD analyses. This is indicated for each of the following figures.

The parasitic drag break-down of all UAVs is shown in Fig. 10. normalized to the reference area of each individual UAV. Total drag varies between 302 and 618 drag counts. This significant margin is in part caused by the normalization with the UAV reference areas that vary considerably. For example, UAV 8 with the highest parasitic drag coefficient is one of the larger UAVs studied here but has a reference wing area that is comparable to the much smaller UAV 4. This increases the drag count for all components of UAV 8 compared to UAVs with larger reference areas.

Comparing the drag break-downs of the analyzed UAVs allows deriving initial characteristic drag aspects for the 


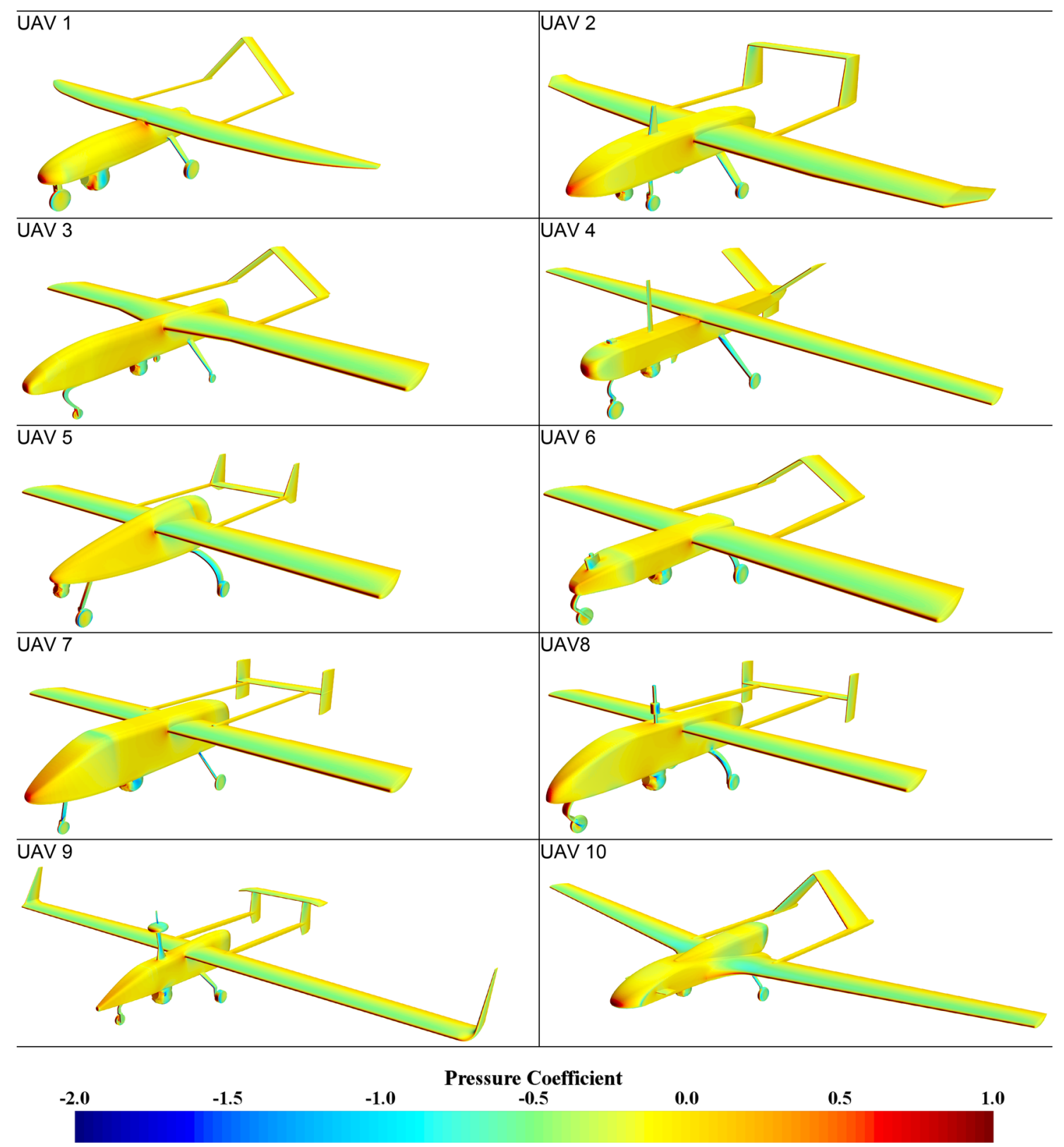

Fig. 9 Isometric views of pressure coefficient on UAV surface (UAVs not to scale)

specific class of SMR UAVs. Wing, tail, fuselage, and tailboom only contribute between 40 and $64 \%$ of the total parasitic drag share, despite having about $90 \%$ of the entire airframe wetted area. Tailboom drag is rather small and does not exceed 3\% of the total parasitic drag. The most important parts of the large airframe components are wing and fuselage. Tail drag is always below $10 \%$ of the total parasitic drag.

The drag of landing gears and EO/IR sensor turrets is very significant for all UAVs, while landing gear drag is higher than turret drag in most instances. Together, landing gear and EO/IR sensor turret are responsible for $36-60 \%$ of the total parasitic drag of an SMR UAV. These components behave like bluff bodies and show high levels of flow separation causing significant pressure drag. Antenna drag does only play a minor role considering the airframe attachments and does never exceed $9 \%$ of the parasitic drag. The antennas of UAVs 8 and 9 show the highest drag share caused by large spherical and cylindrical shapes. However, these were rarely found for the UAVs analyzed in the in-house database, and most UAVs have smaller antennas, causing only minimal parasitic drag.

Research has shown that the parasitic drag break-down is usually specific for an individual aircraft category [25]. Even 


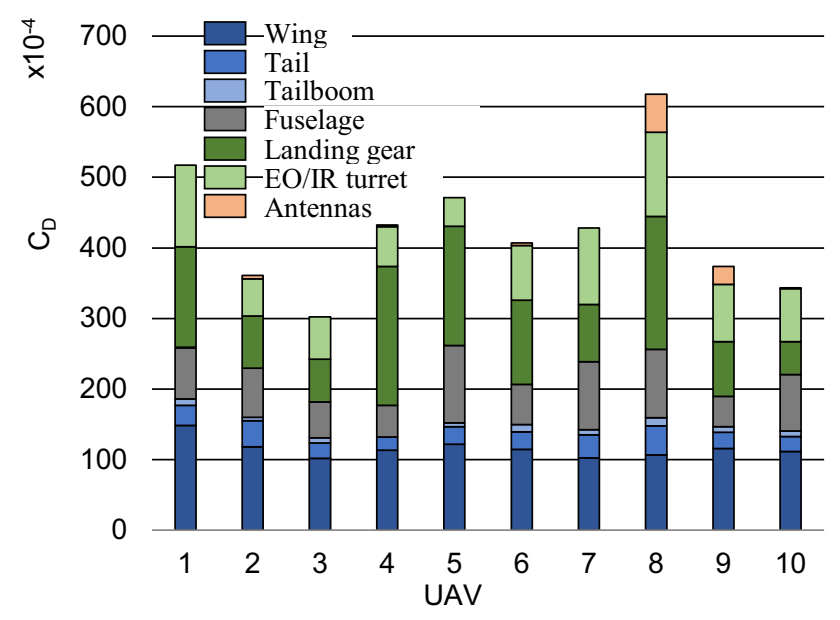

Fig. 10 Parasitic drag break-down (NACA 0015 airfoil on wing)

though the exact drag break-down depends on the individual configuration, clear trends may be observed. The data of all analyzed UAVs were averaged and presents a typical SMR UAV-specific drag break-down, in Fig. 11. This is a common procedure in the statistical analysis of parasitic drag and was also performed for other aircraft categories [25]. An averaged parasitic drag break-down allows focusing on the most critical components that generally cause the highest parasitic drag. Such information is beneficial in early UAV design, as it enables the aerodynamicist to target the right parts for drag reduction measures.

The average SMR UAV wing contributes to $27 \%$ of the total parasitic drag, while the average fuselage shares about $17 \%$ of the total parasitic drag. Both landing gear and turret are responsible for $47 \%$ of an SMR UAV's parasitic drag. On average, the turret of an SMR UAV causes more drag than its fuselage. The drag of antennas and tailbooms is comparably small, and all components together only contribute about $4-5 \%$ of the parasitic drag.

A majority (57-73\%) of total SMR UAV drag is pressure drag mainly caused by the EO/IR sensor turret, landing gear, and fuselage. The comparably high-pressure drag shares of the fuselages are primarily driven by aft separation and manifests themselves as base drag. The friction and pressure drag characteristics of full UAV configurations are, therefore, vastly different compared to larger manned aircraft categories. The parasitic drag of these types of aircraft is mainly friction drag with only a small portion of pressure drag [10]. The specific pressure drag characteristics of SMR UAVs will significantly affect their behaviour considering their equivalent skin friction coefficients.

The averaged SMR UAV-specific parasitic drag breakdown is compared to a typical transport jet drag break-down in Fig. 11. The transport jet drag break down uses data from Ref. [25] showing the parasitic drag in cruise configuration

\section{SMR UAV average}

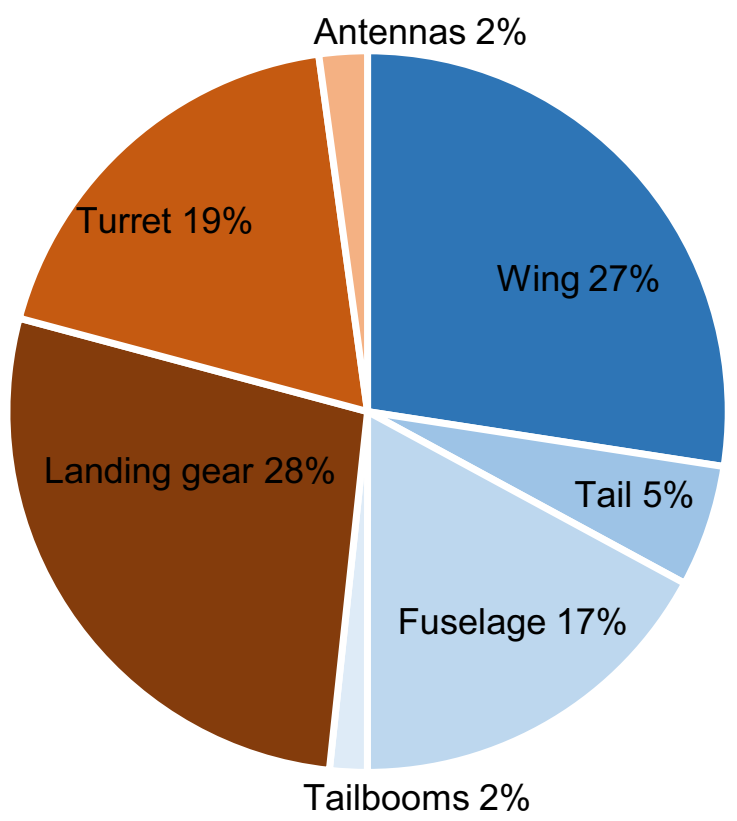

Typical transport aircraft

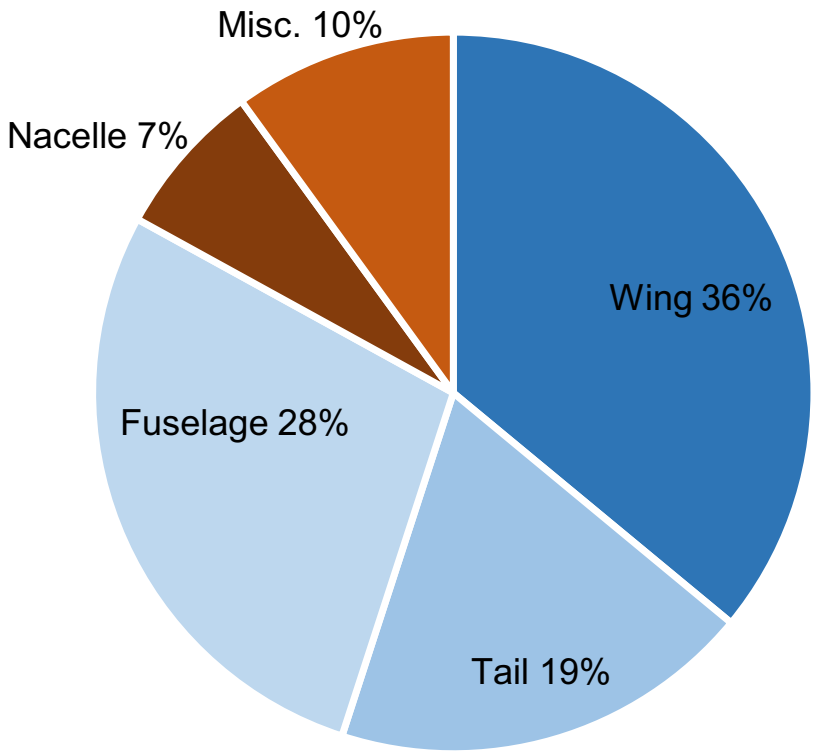

Fig. 11 Averaged SMR UAV parasitic drag break-down (NACA 0015 airfoil on wing) and typical transport drag break-down, with data from Ref. [25]

at Mach 0.8. The differences between UAV and transport aircraft are significant. Wing, tail and fuselage of a transport aircraft are responsible for about $83 \%$ of the parasitic drag, while these surfaces only contribute $53 \%$ to the typical UAV parasitic drag. Transport jets use retractable landing gears due to their focus on speed and range and are usually 
not equipped with EO/IR sensor turrets. Both significantly reduces the parasitic drag and increases the influence of the large airframe surfaces.

The parasitic drag coefficients of all analyzed UAVs are shown in Table 3. They are normalized to the UAV's reference wing area. The equivalent skin friction coefficients of all UAVs are computed according to Eq. (1). The coefficients naturally scatter with the UAV configuration. The table both shows the data from the CFD analyses using a NACA 0015 airfoil on the UAVs' wings and the correction for airfoil camber assuming a NACA 4415 airfoil. The correction for the cambered wing airfoil increases total drag by less than $3 \%$, which directly increases the equivalent skin friction coefficients in the same manner. Such small influence is not relevant given the rather crude accuracy of the method for initial design. The data scatter (standard deviation of 0.00157 ) is on the expectable order of magnitude and also comparable to what is found for other aircraft categories, see Ref. [10].

The distribution of the UAV's equivalent skin friction coefficients is additionally shown in the bar diagram plot of Fig. 12 in comparison to the values for two other aircraft categories given in Ref. [1]. The new SMR UAV equivalent skin friction coefficient is more than double as high compared to the light aircraft—single engine category and more than four times higher compared to the civil transport jet category. The high level of parasitic drag and the significant influence of miscellaneous components are the main reasons for the high equivalent skin friction coefficient of SMR UAVs.

\subsection{Wetted area regressions}

The equivalent skin friction coefficient drag estimation requires knowledge of the new aircraft's wetted area.

Table 3 Equivalent skin friction coefficient of 10 SMR UAVs

\begin{tabular}{lllll}
\hline UAV & $\begin{array}{l}C_{\text {Dmin, }} \\
\text { NACA0015, } \\
\text { drag counts }\end{array}$ & $\begin{array}{l}C_{\text {Dmin, }} \\
\text { corrected for } \\
\text { NACA4415, } \\
\text { drag counts }\end{array}$ & $\begin{array}{l}C_{\text {feq }} \\
\text { NACA0015 }\end{array}$ & $\begin{array}{l}C_{\text {feq }} \\
\text { corrected for } \\
\text { NACA4415 }\end{array}$ \\
\hline 1 & 516.4 & 528.2 & 0.0128 & 0.0131 \\
2 & 360.7 & 370.1 & 0.0099 & 0.0102 \\
3 & 302.2 & 310.4 & 0.0087 & 0.0089 \\
4 & 432.3 & 441.4 & 0.0115 & 0.0118 \\
5 & 470.9 & 506.1 & 0.0124 & 0.0133 \\
6 & 407.0 & 416.2 & 0.0115 & 0.0118 \\
7 & 428.4 & 436.5 & 0.0093 & 0.0095 \\
8 & 617.6 & 626.1 & 0.0132 & 0.0133 \\
9 & 373.7 & 383.0 & 0.0103 & 0.0105 \\
10 & 343.3 & 352.2 & 0.0088 & 0.0090 \\
Average & 425.2 & 437.0 & 0.1084 & 0.01115 \\
\hline
\end{tabular}

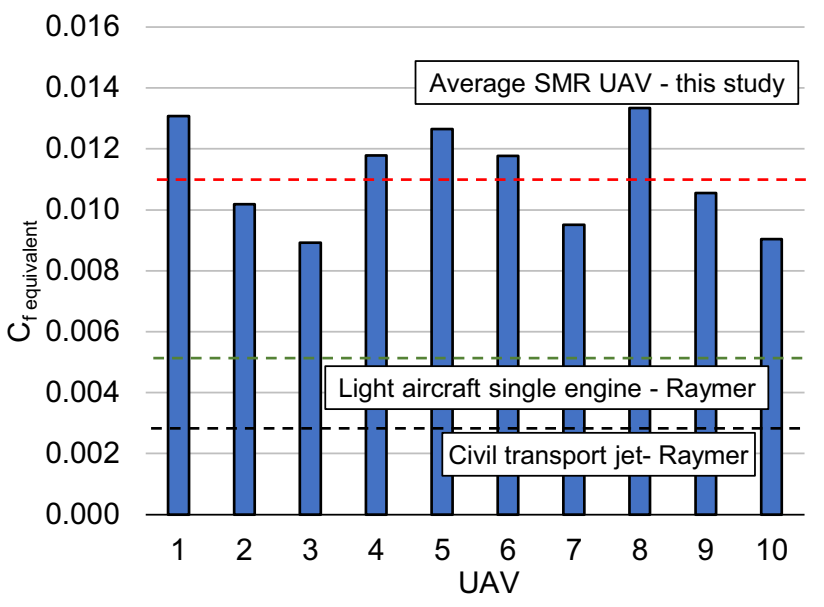

Fig. 12 Equivalent skin friction coefficient of 10 SMR UAVs (corrected for NACA 4415 airfoil on wings)

Depending on the aircraft sizing algorithm and tool, the total wetted area might be calculated based on simple geometrical relationships that are available for the design. However, in case a total wetted area is not available in the design algorithm, several correlations for wetted areas of different aircraft categories are found in the literature (Refs. [1, 10]). However, no relationships for SMR UAVs are available. Therefore, a new correlation between total UAV wetted area and maximum take-off mass is developed using the in-house database. The data is shown in Fig. 13, together with the formulation of a simple power-law fit. The fit can describe the mean of the data; however, scatter is considerable. Whenever

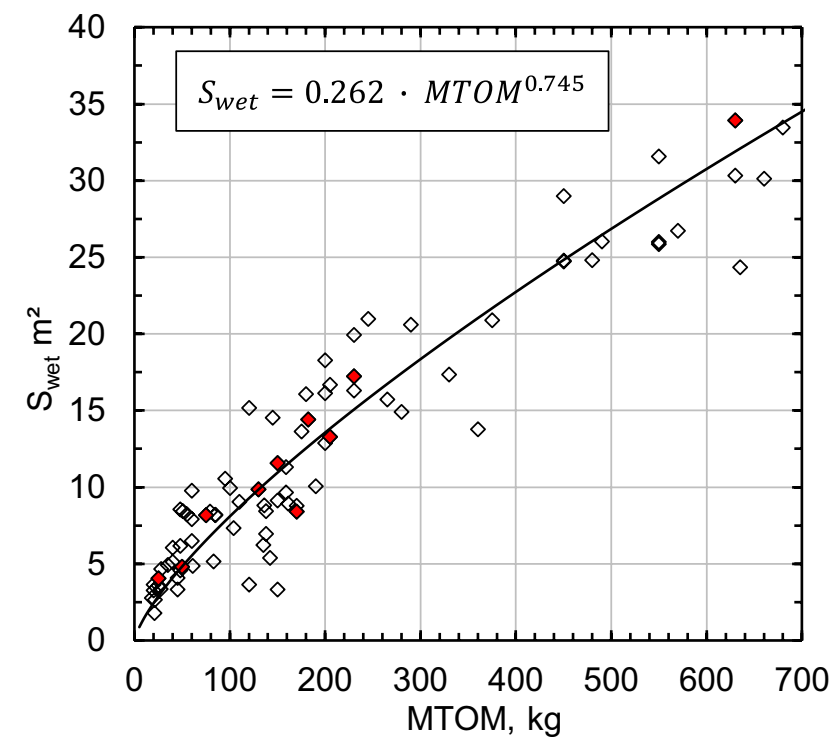

Fig. 13 SMR UAV wetted area vs. MTOM, UAVs used in this study are marked in red 


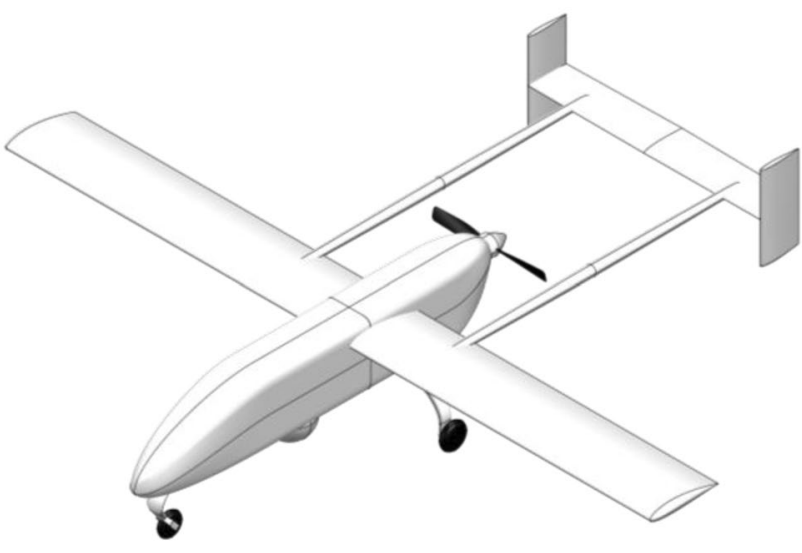

Fig. 14 Notional SMR UAV concept

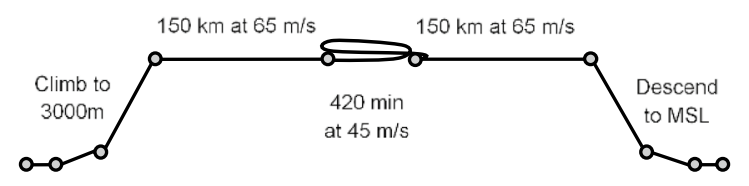

Fig. 15 Notional SMR UAV design mission

more accurate estimations are available, it is highly desirable to use them.

\subsection{UAV sizing process}

To show the impact of the new drag estimation coefficient on the UAV design process, a case study is briefly presented in this section. The author's in-house aircraft design tool [9, $26,27]$ is updated with the newly derived equivalent skin friction coefficient for SMR UAVs. Then, the preliminary sizing of a typical SMR UAV is carried out. The results are compared to the results of a sizing that uses the skin friction coefficients for single-engine prop and for jet aircraft. This study will show how this single parameter will influence the overall design of a UAV, thereby demonstrating its importance.

\subsubsection{Aircraft and mission}

A notional twin-boom reconnaissance SMR UAV is selected for the sizing study. The concept features a single pusher propeller and twin tail booms. A sketch of the notional aircraft is provided in Fig. 14. The UAV is designed for a typical long-endurance surveillance mission, which is shown in Fig. 15. Six hours of loiter at best endurance speed is required, as well as a quick $150 \mathrm{~km}$ dash at $65 \mathrm{~m} / \mathrm{s}$ intoand out of the target area. A $15 \mathrm{~kg}$ payload of surveillance and communication equipment must be carried. Top-level requirements and a mission description are shown in Table 4.

\subsubsection{Sizing approach}

Because this paper focuses on the aerodynamic methods, the sizing approach is not presented in the full extend. The most important points are summarized in this section before the results, and the lessons learned from the results are discussed in more detail in the following section.

The requirements of Sect. 3.3.1 are used to size the aircraft and perform the mass analysis. To assess the impact of the novel equivalent skin friction factor, the sizing process is performed three times. First, the authors' novel skin friction coefficient is used. The sizing is then repeated using Raymer's skin friction coefficient for general aviation aircraft, and once more using the highly optimistic values representative of civil and military jet transports. The authors expect that using these different drag factors will decidedly change the sizing results.

The sizing process is supported by an optimization routine. In particular, a global optimization scheme, the particle swarm method, is employed to find an optimal design for the given set of TLARs and constraints. It is used to select the best possible combination of wing loading W/S,
Table 4 Notional SMR UAV design parameters and mission

\begin{tabular}{llll}
\hline Parameter & Value & Parameter & Value \\
\hline Take-off Ground Roll $(\mathrm{m})$ & 250 & $\begin{array}{l}\text { Sensor } \\
\text { Payload }(\mathrm{kg})\end{array}$ & 15 \\
Rate of Climb at MSL (m/s) & 3 & Taxi and Take-off & at MSL \\
Stall Speed (m/s) & 30 & Climb to Altitude & $2000 \mathrm{~m}$ \\
Dash Speed (m/s) & 65 & Ingress to Target Area & $150 \mathrm{~km}$ \\
Loiter Speed (m/s) & 45 & Loiter over Target Area & for $6 \mathrm{~h}$ \\
Max. Lift Coef. & 1.30 & Egress from Target Area & $150 \mathrm{~km}$ \\
$C_{\mathrm{L}, \text { max }}$ & & & \\
Service & 4600 & Descend, Land, and Taxi & at MSL \\
Ceiling $(\mathrm{m})$ & & & \\
Propulsion & $4-$ Stroke ICE & ICE best BSFC $(\mathrm{g} / \mathrm{kg} / \mathrm{h})$ & 315 \\
System & & & \\
\hline
\end{tabular}


power-to-weight ratio $\mathrm{P} / \mathrm{W}$, wing aspect ratio $\mathrm{AR}$. These design variables have been shown to be appropriate for the initial design of subsonic aircraft [28]. The influence of W/S and AR on the induced drag of the aircraft is captured, using methods from Ref. [1]. However, the zero-lift drag coefficient is calculated using different $C_{\text {feq }}$ and the aircraft's wetted area.

A simplified illustration of the process is provided in Fig. 16. Analysis steps of the sizing process are indicated in blue boxes, while optimization steps are indicated in red boxes. Further information on the design process can be found in Ref. [29]

Minimum MTOM is selected as the optimization's objective. The minimization of MTOM is usually the goal during aircraft design since it is widely acknowledged that "the lightest aircraft that does the job is considered the best" [W. H. Mason, "Modern Aircraft Design Techniques," in Handbook of Transportation Engineering, McGraw-Hill, 2003, pp. 26.1-26.24]. Designers traditionally use aircraft weight to predict cost, as cost scales almost linearly with aircraft weight.

Another suitable measure for assessing aircraft is their energy consumption. The required energy for the design mission is readily available, as it is determined by the mission analysis and then used to determine the aircraft's energy mass fraction. This parameter will also be assessed in the result's analysis.

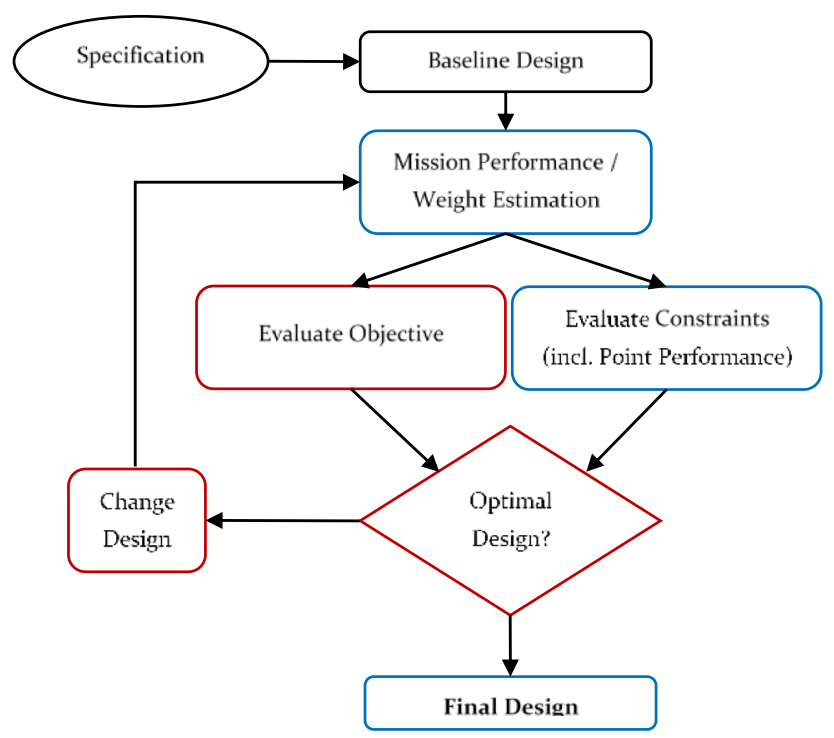

Fig. 16 Optimization process

\subsubsection{Results of SMR UAV Sizing}

The results of the sizing study are presented in Table 5. As expected, using an improper $C_{\text {feq }}$ will drastically impact the sizing and optimization results.

Using the author's $C_{\text {feq }}$ value of 0.01084 (here directly taken from the analysis results using a NACA 0015 as the wing airfoil) resulted in a typical SMR UAV layout: a highly loaded, high AR wing is used to offset the high $C_{\text {Dmin }}$.

If designers were to assume typical $C_{\text {feq }}$ values for light aircraft, the result is driven away from such a typical geometric layout. The optimal AR, as well as the optimal W/S is reduced. Also, $\mathrm{P} / \mathrm{W}$ is reduced, as a lot less aerodynamic drag needs to be overcome. The MTOM is estimated more than $20 \%$ too low, and the energy consumption is off by $61 \%$.

Finally, using a $C_{\text {feq }}$ value of 0.0028 , as is typical for jet transports, drives down AR, W/S, and P/W even further. The result has very little to do with the actual geometry found for SMR UAVs. For this design, MTOM is off by $25 \%$, and energy consumption is $90 \%$ underestimated, compared to the result that uses the author's $C_{\text {feq }}$ value.

These results show that by using an inappropriate $C_{\text {feq }}$, very large errors are introduced in the design process. Such errors are very costly to correct in later design stages. If drag is underestimated for the first sizing computations, a redesign will have to follow once this issue is found. Using the authors' novel $C_{\mathrm{feq}}$ value will prevent additional design iterations.

A scaled illustration of the three different designs is provided in Fig. 17. This makes it easy to appreciate the differences in design that the optimization process selected. It becomes quite clear that selecting a proper drag model as early as possible in the design process is of utmost importance to avoid costly iterations at later stages of a program.

Table 5 Sizing results-notional SMR UAV

\begin{tabular}{|c|c|c|c|}
\hline $\begin{array}{l}C_{\text {feq }} \\
\text { Parameter }\end{array}$ & 0.011 & 0.0055 & $\begin{array}{l}0.0028 \\
\text { Jets }\end{array}$ \\
\hline Parameler & OAVs [autnors] & Lignt Alrcrant & \\
\hline$C_{\text {Dmin }}$ & 0.0470 & 0.0236 & 0.0106 \\
\hline MTOM (kg) & 311 & 256 & 248 \\
\hline Energy (GJ) & 1.74 & 1.08 & 0.92 \\
\hline $\mathrm{W} / \mathrm{S}\left(\mathrm{N} / \mathrm{m}^{2}\right)$ & 714 & 588 & 334 \\
\hline $\mathrm{P} / \mathrm{W}(\mathrm{W} / \mathrm{kg})$ & 110 & 78 & 62 \\
\hline AR & 15.0 & 9.7 & 6.5 \\
\hline \multicolumn{4}{|c|}{ Error relative to UAV $C_{\mathrm{feq}}$ of the authors } \\
\hline MTOM $(\%)$ & - & 21.5 & 25.4 \\
\hline Energy $(\%)$ & - & 61.1 & 89.1 \\
\hline
\end{tabular}




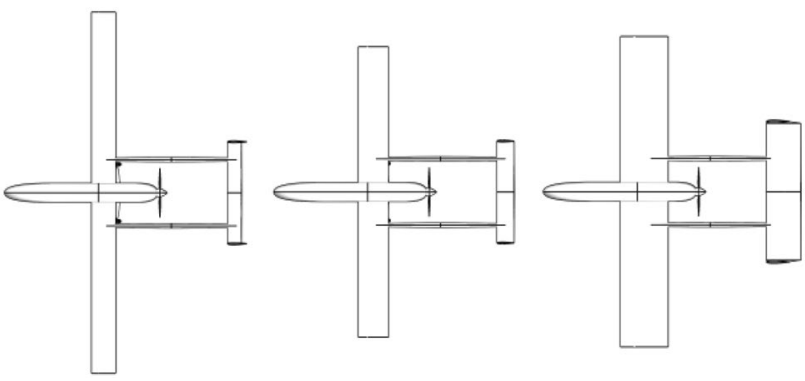

Fig. 17 Top-view of the three sizing results $C_{\text {feq }}=0.011 ; 0.0055$; 0.0028 from left to right

\section{Conclusion}

The paper presents the derivation of a new equivalent skin friction coefficient for estimating the parasitic drag of SMR UAVs. Such UAVs are nowadays employed on surveillance, reconnaissance, and search and rescue missions. Their parasitic drag is much larger compared to other aircraft categories. It is significantly influenced by the presence of miscellaneous components like fixed landing gears and EO/IR sensor turrets. The new equivalent skin friction coefficient is more than double as high as common coefficients found for small single-engine aircraft. The coefficient might be employed with a new wetted area regression for SMR UAVs that allows determining the wetted area based on a UAVs maximum take-off mass.

The initial sizing of a novel SMR UAV is significantly affected by the parasitic drag estimation. Choosing an incorrect friction coefficient will drive the design significantly away from an optimal layout. The new equivalent skin friction coefficient significantly enhances drag estimation in early conceptual design stages.

Acknowledgements The authors like to express their gratitude to Siemens PLM Software for providing academic licenses of their software StarCCM+.

Funding Open Access funding enabled and organized by Projekt DEAL. Funding for this work was provided by the Department of Aerospace Engineering of FH Aachen University of Applied Sciences and the College of Science Engineering and Health of the Royal Melbourne Institute of Technology.

Open Access This article is licensed under a Creative Commons Attribution 4.0 International License, which permits use, sharing, adaptation, distribution and reproduction in any medium or format, as long as you give appropriate credit to the original author(s) and the source, provide a link to the Creative Commons licence, and indicate if changes were made. The images or other third party material in this article are included in the article's Creative Commons licence, unless indicated otherwise in a credit line to the material. If material is not included in the article's Creative Commons licence and your intended use is not permitted by statutory regulation or exceeds the permitted use, you will need to obtain permission directly from the copyright holder. To view a copy of this licence, visit http://creativecommons.org/licenses/by/4.0/.

\section{References}

1. Raymer, D.P.: Aircraft design. A conceptual approach, 6th edn. AIAA Education Series, Reston (2019) ISBN 978-1-62410-490-9

2. Valavanis, K.P., Vachtsevanos, G.J. (eds.): Handbook of unmanned aerial vehicles. Springer, Dordrecht (2015) ISBN 978-90-481-9706-4

3. Austin, R.: Unmanned aircraft systems. UAVS design, development and deployment, 2nd edn. Wiley, Chichester (2010) ISBN 978-0-470-05819-0

4. Sadraey, M.H.: Unmanned aircraft design. A review of fundamentals, Morgan \& Claypool Publishers (2017) ISBN 9781681731681

5. Gundlach, J.: Designing unmanned aircraft systems: a comprehensive approach, 2nd edn. AIAA Education Series, Reston (2014) ISBN 978-1-62410-261-5

6. Panagiotou, P., Yakinthos, K.: Aerodynamic efficiency and performance enhancement of fixed-wing UAVs. Aerosp. Sci. Technol. (2019). https://doi.org/10.1016/j.ast.2019.105575

7. Panagiotou, P., Kaparos, P., Salpingidou, C., Yakinthos, K.: Aerodynamic design of a MALE UAV. Aerosp. Sci. Technol. 50, 127-138 (2016). https://doi.org/10.1016/j.ast.2015.12.033

8. Götten, F., Havermann, M., Braun, C., Gómez, F., Bil, C.: On the applicability of empirical drag estimation methods for unmanned air vehicle design. In: $18^{\text {th }}$ AIAA Aviation Technology Integration and Operations Conference. AIAA, Reston (2018) https://doi.org/ 10.2514/6.2018-3192.

9. Finger, D.F., Bil, C., Braun, C.: Initial sizing methodology for hybrid-electric general aviation aircraft. J. Aircr (2019). https:// doi.org/10.2514/1.C035428

10. Roskam, J.: Airplane Design I-VIII. Roskam Aviation and Engineering Corporation, Ottawa (1987)

11. Götten, F., Finger, D.F., Braun, C., Havermann, M., Bil, C., Gómez, F.: Empirical correlations for geometry build-up of fixed wing unmanned air vehicles. In: The Proceedings of the 2018 Asia-Pacific International Symposium on Aerospace Technology (APISAT 2018), pp. 1365-1381. Springer, Singapore (2019) https://doi.org/10.1007/978-981-13-3305-7_109

12. Gloudemans, J., Davis, P., Gelhausen, P.: A rapid geometry modeler for conceptual aircraft. In: 34th Aerospace Sciences Meeting and Exhibit. AIAA, Reston (1996) https://doi.org/10.2514/6. 1996-52.

13. Marongiu, C., Tognaccini, R., Ueno, M.: Lift and lift-induced drag computation by lamb vector integration. AIAA J. 51(6), 1420-1430 (2013). https://doi.org/10.2514/1.J052104

14. Takahashi, T., Ou, C.-W.: When higher fidelity models degrade our understanding of induced drag-the tragedy of the trefftz plane integral. AIAA Scitech 2020 Forum (2020). https://doi.org/ 10.2514/6.2020-0276

15. CD-adapco, Validation of Star-CCM+ for External Aerodynamics in the Aerospace Industry https://mdx2.plm.automation.siemens. $\mathrm{com} /$ sites/default/files/Presentation/CD-adapco_AeroValidation_ v7.pdf-aerospace-industry. Retrieved 22 Jan 2020

16. Hirsch, C.: Computational methods for inviscid and viscous flows. Wiley, Chichester (2002) ISBN 0471924520

17. Goetten, F., Finger, D.F., Marino, M., Cees, B., Havermann, M., Braun, C.: A review of guidelines and best practices for subsonic aerodynamic simulations using RANS CFD. In: APISAT 2019 Asia Pacific International Symposium on Aerospace Technology, pp. 227-245 (2019) ISBN 9781925627404

18. Hellsten, A.K.: New advanced k-w turbulence model for high-lift aerodynamics. AIAA J. 43(9), 1857-1869 (2005). https://doi.org/ 10.2514/1.13754

19. Spalart, P.R.: Strategies for turbulence modeling and simulations. Int. J. Heat Fluid Flow 21(3), 252-263 (2000). https://doi.org/10. 1016/S0142-727X(00)00007-2 
20. Spalart, P.R., Rumsey, C.L.: Effective inflow conditions for turbulence models in aerodynamic calculations. AIAA J. 45(10), 2544-2553 (2007). https://doi.org/10.2514/1.29373

21. Lienhard, J.H.: Synopsis of lift, drag, and vortex frequency data for rigid circular cylinders. Bulletin 300 Washington State University, Pullman (1966)

22. Bray, R.M.: A wind tunnel study of the Pioneer remotely piloted vehicle. Naval Postgraduate School, Monterey (1991) http://hdl. handle.net/10945/43784. Retrieved 4 May 2020

23. Young, A.D.: Boundary Layers. AIAA Education Series, Washington, DC (1989) ISBN 0930403576

24. Abbott, I.H., von Doenhoff, A.E. von, Stivers, L.S.: Summary of Airfoil Data. NACA TR-824 National Advisory Committee for Aeronautics, Langley Field (1945)

25. Jobe, C.E.: Prediction of Aerodynamic Drag. AFWAL-TM-84-203 U.S. Air Force, Dayton, Ohio (1984)

26. Finger, D.F., Götten, F., Braun, C., Bil, C.: Mass, primary energy, and cost. The impact of optimization objectives on the initial sizing of hybrid-electric general aviation aircraft. CEAS Aeronaut. J. 18(2), 76 (2020). https://doi.org/10.1007/s13272-020-00449-8

27. Finger, D.F., de Vries, R., Vos, R., Braun, C., Bil, C.: A Comparison of Hybrid-Electric Aircraft Sizing Methods. AIAA Scitech 2020 Forum (2020)

28. Raymer, D.P.: Enhancing Aircraft Conceptual Design using Multidisciplinary Optimization. PhD Thesis, Stockholm (2002) ISBN 91-7283-259-2

29. Finger, D.F.: Methodology for Multidisciplinary Aircraft Design under Consideration of Hybrid-Electric Propulsion Technology. $\mathrm{PhD}$ Thesis Royal Melbourne Institute of Technology, Melbourne (2020) https://doi.org/10.13140/RG.2.2.31468.59523

Publisher's Note Springer Nature remains neutral with regard to jurisdictional claims in published maps and institutional affiliations. 